\title{
Uso da combinação linear de soluções físicas no estudo da natureza do ponto estacionário da ação
}

\author{
The use of the linear combination of physical solutions in the study of the \\ nature of the stationary point of action
}

\author{
Fábio Pascoal dos Reis ${ }^{1}$ \\ Uilian de Oliveira Pereira ${ }^{2}$ \\ Pablo Henrique Menezes ${ }^{3}$ \\ Elisângela Aparecida Y. Castro ${ }^{4}$
}

\begin{abstract}
Resumo. O princípio de Hamilton afirma que, dentre todas as curvas entre dois extremos fixos, o caminho que é realmente seguido por um sistema físico será aquele que atribui um valor estacionário (pontos mínimos, máximos ou de sela) à ação (uma integral no tempo da diferença entre a energia cinética e a energia potencial, tomada entre o tempo inicial e o tempo final em que sistema funciona). É comum usar uma ferramenta matemática chamada Cálculo de Variacional para estudar o princípio de Hamilton. O Cálculo Variacional lida com funcionais (funções das funções) e é uma versão mais geral e mais complexa do cálculo usual que aprendemos na graduação. Neste artigo, apresentamos uma abordagem alternativa e mais simples ao estudo do princípio de Hamilton. Estudamos a natureza do movimento estacionário da ação de três sistemas físicos: partícula livre, lançamento vertical e oscilador harmônico, usando como movimento virtual uma combinação linear da solução física desses três sistemas. Encontramos evidências de que a solução física do problema de partículas livres leva a um mínimo em sua ação. Os mesmos resultados ocorrem no problema de lançamento vertical. A solução física do oscilador harmônico leva a um ponto mínimo ou de sela em sua ação, a depender do intervalo de tempo de funcionamento do sistema.
\end{abstract}

\footnotetext{
${ }^{1}$ Instituto de Ciências Exatas e Naturais do Pontal, Universidade Federal de Uberlândia, fabiopr@ufu.br

${ }^{2}$ Instituto de Ciências Exatas e Naturais do Pontal, Universidade Federal de Uberlândia, uilianop@gmail.com

${ }^{3}$ Instituto de Ciências Exatas e Naturais do Pontal, Universidade Federal de Uberlândia, pablohenriquemenezes11@hotmail.com

${ }^{4}$ Instituto de Ciências Exatas e Naturais do Pontal, Universidade Federal de Uberlândia, elisangela.castro@ufu.br
} 
Palavras-chave. Mecânica clássica, ação, princípio de Hamilton, cálculo funcional.

\begin{abstract}
Hamilton's principle states that among all curves between two end points, the path actually followed by a physical system will always be the one that gives an stationary value (minimum, maximum or a saddle points) to the Hamilton's action (an integral over time of the difference between the kinetic and the potential energy, taken between the initial time and the final time of the development of the system). It is common to use a mathematical tool called Calculus of Variations to study the Hamilton's principle. The Calculus of Variations deals with functionals (functions of functions) and is a more general and more complex version of the usual calculus that we learn in university. In this paper we present an alternative, and simpler, approach to the study of the Hamilton's principle. We study the nature of the action's stationary space-time trajectory of three physical systems: free-particle, vertical launch and harmonic oscillator, using as virtual motion a linear combination of the physical solution of this three systems. We find evidences that the physical solution of the free-particle problem leads to a minimum on its action. The same results occours on the vertical launch problem. The physical solution of the harmonic oscillator leads to a minimum or a saddle point on its action deppending on the time interval of the development of the system.
\end{abstract}

Keywords. Classical mechanics, action, Hamilton's principle, functional calculus. 


\section{Introdução}

Considere uma partícula cujo movimento unidimensional real é descrito pela função $q_{r}(t)$. O princípio de Hamilton estabelece que $q_{r}(t)$ (durante o intervalo de tempo $t_{i} \leq t \leq t_{f}$ ) é tal que a grandeza física chamada ação, definida pelo funcional

$$
\mathcal{S}[q]=\int_{t_{i}}^{t_{f}} d t \mathcal{L}(q, \dot{q} ; t)
$$

apresenta um valor estacionário, se comparado a todas as trajetórias virtuais com as mesmas configurações iniciais e finais. Em $(1), \mathcal{L}(q, \dot{q} ; t)$ é a lagrangeana do sistema que é definida como

$$
\mathcal{L}(q, \dot{q} ; t)=\frac{m \dot{q}^{2}}{2}-V(q),
$$

onde $V(q)$ é a energia potencial do sistema.

Analogamente ao cálculo diferencial, a derivada funcional de $\mathcal{S}$ é definida como

$$
D_{\eta} \mathcal{S}[q]=\frac{\mathcal{S}[q+\epsilon \eta]-\mathcal{S}[q]}{\epsilon}
$$

onde a função desvio $\eta$ é uma função arbitrária com $\eta\left(t_{i}\right)=\eta\left(t_{f}\right)=0$ e $\epsilon$ é uma constante infinitesimal e adimensional. A função que gera o valor estacionário é obtida ao procurar qual função $q_{r}$ leva a uma derivada funcional de $\mathcal{S}$ nula. Seguindo esse procedimento, chega-se a equação de Euler-Lagrange (veja por exemplo [1] e [2])

$$
\frac{d}{d t}\left(\frac{\partial \mathcal{L}}{\partial \dot{q}_{r}}\right)-\frac{\partial \mathcal{L}}{\partial q_{r}}=0
$$

Vale destacar que este resultado independe da função desvio.

Embora também seja conhecido como princípio de mínima ação, a ação apresentará um mínimo, um máximo ou um "ponto"de sela, quando avaliada na trajetória que será percorrida pela partícula. Analogamente ao que ocorre no cálculo diferencial, essa classificação dependeria do sinal das derivadas segundas do funcional $\mathcal{S}$.

No artigo When action is not least, publicado em 2007, C.G. Gray e E.F. Taylor apresentam uma discussão detalhada sobre o sinal da derivada segunda da ação [3]. Nele, os autores estudam o caso em que a energia cinética é quadrática na velocidade e a energia potencial depende da posição e do tempo. Fica claro neste artigo que, em certos casos, para determinar o sinal da derivada segunda, é necessário especificar a forma da função desvio $\eta$ e extrapolar o resultado para uma função desvio arbitrária.

Em A derivada funcional de segunda ordem da ação: investigando minimalidade, 
maximalidade e "ponto"sela, publicado em 2012, W.H.C. Freire generaliza o resultado de C.G. Gray e E.F. Taylor, levando em consideração um potencial dependente da posição, do tempo e da velocidade [4].

Em Condition for minimal harmonic oscillator action, publicado em 2017, M. Moriconi estuda especificamente a ação do oscilador harmônico [5]. Neste trabalho, utiliza-se uma função desvio da forma $\eta(t)=u(t) \sin \left(\pi t /\left(t_{f}-t_{i}\right)\right)$ para calcular uma expressão para $\mathcal{S}$. A expressão final para a ação corresponde a soma de duas parcelas: uma relacionada apenas ao movimento real e outra relacionada apenas ao desvio. Depois disso, analisa-se a parcela da ação gerada exclusivamente pelo desvio para mostrar que para um intervalo de tempo $t_{f}-t_{i}$ suficientemente curto, $q_{r}$ minimiza $\mathcal{S}$. Por outro lado, quando o intervalo de tempo $t_{f}-t_{i}$ é suficientemente longo, $q_{r}$ é um "ponto"de sela de $\mathcal{S}$. Essa condição foi, também, obtida nos dois artigos citados anteriormente.

Nosso principal objetivo, nesse artigo, é apresentar uma abordagem alternativa que possa contribuir didaticamente para uma melhor compreensão do Princípio de Hamilton. Nos restringiremos a analisar a ação usando uma família específica de movimentos virtuais: a combinação linear de três movimentos reais com extremos fixos. Perdemos em generalidade, mas ganhamos em simplicidade, uma vez que nessa abordagem, a ação passa a ser escrita como uma função de duas variáveis, no lugar de um funcional.

\section{Movimentos reais e virtuais}

O movimento do problema da partícula livre é descrito pela equação

$$
q_{1}=\frac{q_{f} t}{\tau}
$$

onde usamos as condições de contorno $q_{1}(0)=0$ e $q_{1}(\tau)=q_{f}$. A solução do lançamento vertical pode ser escrita da forma

$$
q_{2}=\frac{q_{f} t}{\tau}+\frac{g t(\tau-t)}{2}
$$

Já a do oscilador harmônico é dada por

$$
q_{3}=\frac{q_{f} \sin (\omega t)}{\sin (\omega \tau)} .
$$

As funções $q_{1}, q_{2}$ e $q_{3}$ podem ser obtidas usando, respectivamente, $V=0, V=m g q$ e $V=m \omega^{2} q^{2} / 2$ na lagrangeana (2) e resolvendo a equação diferencial de segundo grau resultante da equação de Euler-Lagrange. 
Em nossos estudos, utilizaremos a família de soluções virtuais dadas por

$$
q_{v}=(1-\alpha-\beta) q_{1}+\beta q_{2}+\alpha q_{3}
$$

Como as funções $q_{1}, q_{2}$ e $q_{3}$ assumem os mesmos valores nos extremos, a combinação linear descrita acima também terá esses mesmos valores nos extremos. Além disso, $q_{v}$ representa as soluções do problema da partícula livre quando $\alpha=0$ e $\beta=0$, do lançamento vertical quando $\alpha=0$ e $\beta=1$ e do oscilador harmônico quando $\alpha=1$ e $\beta=0$. Vale ressaltar que $q_{1}, q_{2}$ e $q_{3}$ não formam uma base completa e, portanto, por mais que variemos os parâmetros $\alpha$ e $\beta$ em $q_{v}$, não é possível varrer todos os movimentos virtuais possíveis com esses mesmos extremos fixos.

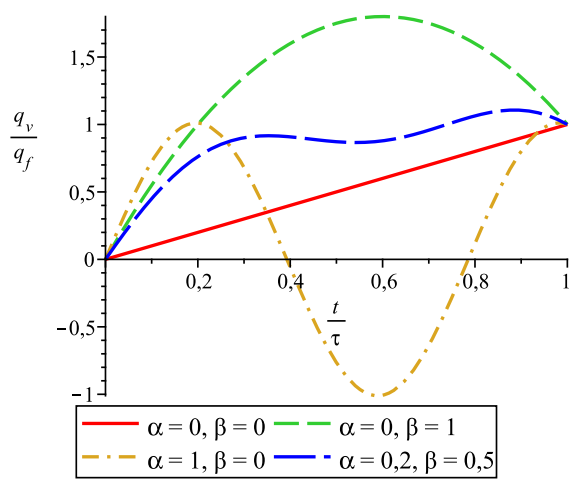

Figura 1: Gráfico de $q_{v} / q_{f}$ em função de $t / \tau$. Neste gráfico, usamos $g \tau^{2} / q_{f}=1 \mathrm{e}$ $\omega \tau=8$.

\section{Ação do problema da partícula livre}

Usando $V=0$ e (6) em (2) e (1), obtemos a ação do problema da partícula livre avaliada em $q_{v}$ :

$$
\begin{aligned}
\mathcal{S}_{1}(\alpha, \beta)= & (1-\alpha-\beta)^{2} \mathcal{T}_{11}+\beta^{2} \mathcal{T}_{22}+\alpha^{2} \mathcal{T}_{33} \\
& +2(1-\alpha-\beta) \beta \mathcal{T}_{12}+2(1-\alpha-\beta) \alpha \mathcal{T}_{13} \\
& +2 \alpha \beta \mathcal{T}_{23},
\end{aligned}
$$

onde definimos

$$
\mathcal{T}_{i j}=\frac{m}{2} \int_{0}^{\tau} \dot{q}_{i} \dot{q}_{j} d t
$$

Fixando uma das velocidades como sendo a da partícula livre, que é uma constante, $\dot{q}_{1}$ pode ser retirada de dentro da integral (8), restando somente como integrando $\dot{q}_{j}$. A inte- 
gral de $\dot{q}_{j}$ avaliada em $t=0$ e $t=\tau$, para qualquer valor de $j$ será 0 e $q_{f}$, respectivamente. Desta forma,

$$
\mathcal{T}_{1 i}=\frac{m q_{f}^{2}}{2 \tau} .
$$

Usando a relação (9) podemos reescrever a ação (7) como

$$
\begin{aligned}
\mathcal{S}_{1}(\alpha, \beta)= & \mathcal{T}_{11}+\alpha^{2}\left(\mathcal{T}_{33}-\mathcal{T}_{11}\right)+\beta^{2}\left(\mathcal{T}_{22}-\mathcal{T}_{11}\right) \\
& +2 \alpha \beta\left(\mathcal{T}_{23}-\mathcal{T}_{11}\right)
\end{aligned}
$$

As outras componentes de $\mathcal{T}_{i j}$ também pode ser calculadas sem muita dificuldade, utilizando integração direta, integração por partes e substituição trigonométrica. Listamos os resultados a seguir:

$$
\begin{aligned}
& \mathcal{T}_{22}=\frac{m q_{f}^{2}}{2 \tau}+\frac{m g^{2} \tau^{3}}{24} \\
& \mathcal{T}_{23}=\frac{m q_{f}^{2}}{2 \tau}-\frac{m g q_{f} \tau}{4}+\frac{m g q_{f}}{2 \omega \sin (\omega \tau)}-\frac{m g q_{f} \cos (\omega \tau)}{2 \omega \sin (\omega \tau)} \\
& \mathcal{T}_{33}=\frac{m q_{f}^{2} \omega \cos (\omega \tau)}{4 \sin (\omega \tau)}+\frac{m q_{f}^{2} \omega^{2} \tau}{4 \sin ^{2}(\omega \tau)}
\end{aligned}
$$

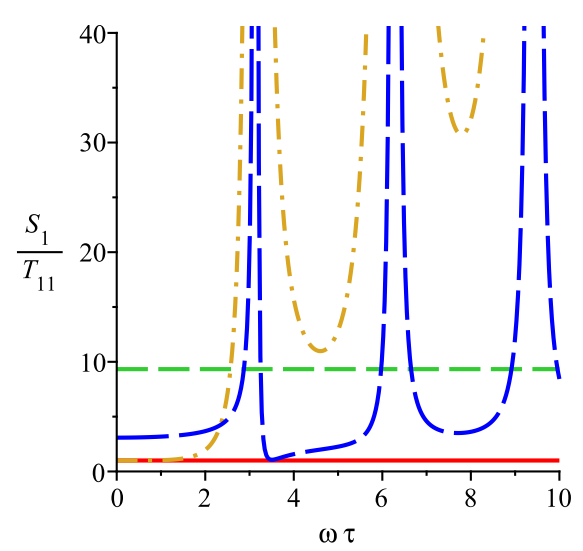

Figura 2: Gráfico de $\mathcal{S}_{1} / \mathcal{T}_{11}$ em função de $\omega \tau$. Usamos $g \tau^{2} / q_{f}=1$ e os mesmos padrões da Fig. 1.

Na Figura 2, apresentamos o gráfico normalizado da ação em função do tempo para diferentes combinações de valores de $\alpha$ e $\beta$. Como podemos ver, das combinações ilustradas, a ação da partícula livre avaliada em seu movimento real $(\alpha=\beta=0)$ é a que apresenta o menor valor para todos instantes de tempo. Isto é um indício de que o mo- 
vimento real da partícula livre minimiza a ação. Agora, pensaremos em $\mathcal{S}_{1}$ como uma função de duas variáveis e, em seguida, discutiremos como encontrar seus pontos estacionários e classificá-los.

O ponto estacionário de $\mathcal{S}_{1}$ é obtido procurando pelos valores de $\alpha$ e $\beta$, que tornam nulas as derivadas dessa ação em função desses dois parâmetros. Assim, o valor estacionário de $\mathcal{S}_{1}$ é atingido quando $\alpha=\beta=0$.

Para estudar a natureza do ponto estacionário, é necessário analisar as derivadas parciais de segunda ordem avaliadas neste mesmo ponto. Aplicando resultados de [6], definimos as funções teste

$$
\begin{aligned}
& D_{1}=\frac{\partial^{2} \mathcal{S}}{\partial \beta^{2}} \\
& D_{2}=\left(\frac{\partial^{2} \mathcal{S}}{\partial \beta^{2}} \frac{\partial^{2} \mathcal{S}}{\partial \alpha^{2}}\right)\left(\frac{\partial^{2} \mathcal{S}}{\partial \alpha \partial \beta}\right)^{-2}
\end{aligned}
$$

Se,

- $D_{1}>0$ e $D_{2}>1$, o ponto estacionário é um mínimo;

- $D_{1}<0$ e $D_{2}>1$, o ponto estacionário é um máximo;

- $D_{2}<1$, o ponto estacionário é um ponto de sela.

No caso da partícula livre, ao usarmos (10), obtemos $D_{1}=2\left(\mathcal{T}_{22}-\mathcal{T}_{11}\right)$. Substituindo (9) e (11), é possível mostrar que

$$
D_{1}=\frac{m g^{2} \tau^{3}}{12}>0
$$

Usando (9), (10) e (11), pode-se mostrar que

$$
D_{2}=\frac{(\omega \tau)^{2}}{6} \frac{\left(\omega \tau \sin (\omega \tau) \cos (\omega \tau)+(\omega \tau)^{2}-2 \sin ^{2}(\omega \tau)\right)}{(\omega \tau \sin (\omega \tau)+2 \cos (\omega \tau)-2)^{2}}
$$

Chama a atenção que $D_{2}$ não depende de $q_{f}, m$ e $g$.

A função teste $D_{2}$ tem uma forma mais complexa que $D_{1}$, mas como podemos ver em Fig. 3, ela é sempre positiva e maior que 1. Esses resultados confirmam que, dentre todos os movimentos virtuais abrangidos pela família (6), o movimento real da partícula livre $(\alpha=\beta=0)$ é um ponto de mínimo de $\mathcal{S}_{1}$ para quaisqer valores de $\tau$, $\omega$ e $q_{f}$. Em [3] e [4], os autores indicam que a ação no problema da partícula livre é minimizada quando avaliada em seu movimento real, portanto, nosso resultado está de acordo com o estabelecido na literatura. 


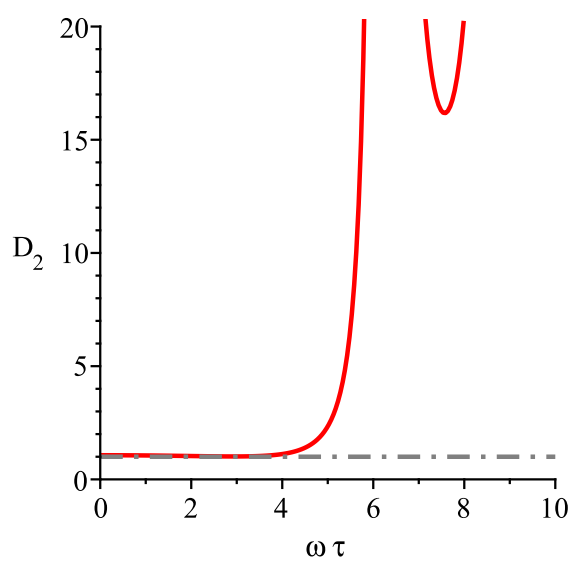

Figura 3: Gráfico de $D_{2}$ em função de $\omega \tau$. A linha traço-ponto representa $D_{2}=1$.

\section{Ação do lançamento vertical}

A ação do lançamento vertical avaliada em $q_{v}$ é dada por

$$
\begin{aligned}
\mathcal{S}_{2}(\alpha, \beta)= & \mathcal{T}_{11}-\mathcal{V}_{1}+\alpha^{2}\left(\mathcal{T}_{33}-\mathcal{T}_{11}\right) \\
& +\beta^{2}\left(\mathcal{T}_{22}-\mathcal{T}_{11}\right)+2 \alpha \beta\left(\mathcal{T}_{23}-\mathcal{T}_{11}\right) \\
& +\alpha\left(\mathcal{V}_{1}-\mathcal{V}_{3}\right)+\beta\left(\mathcal{V}_{1}-\mathcal{V}_{2}\right)
\end{aligned}
$$

onde

$$
\mathcal{V}_{i}=m g \int_{0}^{\tau} d t q_{j} .
$$

Integrando por partes e usando que $\ddot{q}_{2}=-g$, é possível mostrar que

$$
\mathcal{T}_{2 j}-\mathcal{T}_{11}=\frac{\mathcal{V}_{j}-\mathcal{V}_{1}}{2}
$$

o que nos leva a reescrever a ação como

$$
\begin{aligned}
\mathcal{S}_{2}(\alpha, \beta)= & \mathcal{T}_{22}-\mathcal{V}_{2}+\alpha^{2}\left(\mathcal{T}_{33}-\mathcal{T}_{11}\right) \\
& +(\beta-1)^{2}\left(\mathcal{T}_{22}-\mathcal{T}_{11}\right) \\
& +2 \alpha(\beta-1)\left(\mathcal{T}_{23}-\mathcal{T}_{11}\right) .
\end{aligned}
$$

Já calculamos $\mathcal{T}_{i j}$ na seção anterior, logo, falta apenas calcular $\mathcal{V}_{2}$ para termos a expressão completa de $\mathcal{S}_{2}$. Usando (4) em (12) e realizando uma integração simples, obtemos

$$
\mathcal{V}_{2}=\frac{m g q_{f} \tau}{2}+\frac{m g^{2} \tau^{3}}{12}
$$


Na Figura 4, apresentamos o gráfico normalizado da ação do lançamento vertical em função do tempo para diferentes combinações de valores de $\alpha$ e $\beta$. Nas combinações ilustradas, a ação do lançamento vertical avaliada em seu movimento real ( $\alpha=0$ e $\beta=1$ ) apresentou o menor valor para todos instantes de tempo.

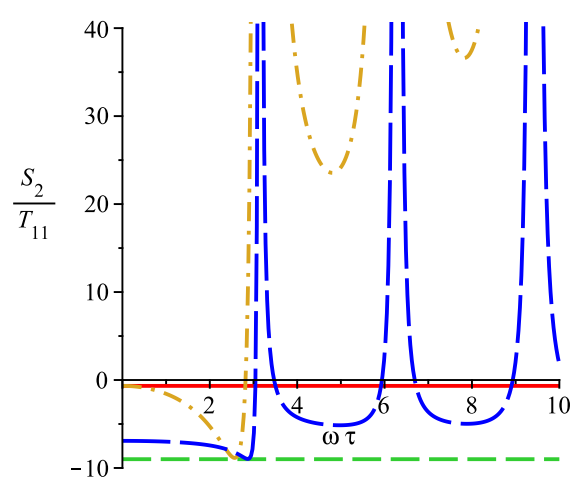

Figura 4: Gráfico de $\mathcal{S}_{2} / \mathcal{T}_{11}$ em função de $\omega \tau$. Usamos $g \tau^{2} / q_{f}=1$ e os mesmos padrões da Fig. 1.

A ação $\mathcal{S}_{2}$ apresenta derivadas parciais em função de $\alpha$ e $\beta$ nulas, quando $\alpha=0$ e $\beta=1$. Isso implica que, como esperado, a função de movimento real do lançamento vertical leva sua ação a um valor estacionário. As derivadas parciais de segunda ordem de $\mathcal{S}_{2}$ são as mesmas de $\mathcal{S}_{1}$, portanto, assim como no caso da partícula livre, dentre todos os movimentos virtuais abrangidos pela família (6), o movimento real do lançamento vertical ( $\alpha=0$ e $\beta=1$ ) é o que leva ao menor valor da ação $\mathcal{S}_{2}$. Mais uma vez, nossos resultados estão de acordo com os gráficos apresentados em [3] e [4].

\section{Ação do oscilador harmônico}

A ação do oscilador harmônico é dada por

$$
\begin{aligned}
\mathcal{S}_{3}(\alpha, \beta)= & (1-\alpha-\beta)^{2}\left(\mathcal{T}_{11}-\mathcal{V}_{11}\right)+\beta^{2}\left(\mathcal{T}_{22}-\mathcal{V}_{22}\right) \\
& +\alpha^{2}\left(\mathcal{T}_{33}-\mathcal{V}_{33}\right)+2(1-\alpha-\beta) \beta\left(\mathcal{T}_{12}-\mathcal{V}_{12}\right) \\
& +2(1-\alpha-\beta) \alpha\left(\mathcal{T}_{13}-\mathcal{V}_{13}\right)+2 \alpha \beta\left(\mathcal{T}_{23}-\mathcal{V}_{23}\right)
\end{aligned}
$$

onde

$$
\mathcal{V}_{i j}=\frac{m \omega^{2}}{2} \int_{0}^{\tau} d t q_{i} q_{j}
$$


Fazendo a integral por partes de $\mathcal{T}_{3 j}$, usando que $\ddot{q}_{3}=-\omega^{2} q_{3}$ e substituindo (5), obtemos a relação

$$
\mathcal{T}_{3 j}-\mathcal{V}_{3 j}=\frac{\omega q_{f}^{2} \cos (\omega \tau)}{\sin (\omega \tau)}
$$

Usando (9) e (14), podemos reescrever a expressão da ação da seguinte forma

$$
\begin{aligned}
\mathcal{S}_{3}(\alpha, \beta)= & \mathcal{T}_{33}-\mathcal{V}_{33}+(\alpha-1)^{2}\left(\mathcal{V}_{13}-\mathcal{V}_{11}\right) \\
& +\beta^{2}\left(\mathcal{T}_{22}-\mathcal{T}_{11}+2 \mathcal{V}_{12}-\mathcal{V}_{11}-\mathcal{V}_{22}\right) \\
& +2(\alpha-1) \beta\left(\mathcal{V}_{12}-\mathcal{V}_{11}\right)
\end{aligned}
$$

As componentes de $\mathcal{V}_{i j}$ também podem ser obtidas explicitamente. Resolvendo as integrais, obtemos

$$
\begin{aligned}
& \mathcal{V}_{11}=\frac{m q_{f}^{2} \omega^{2} \tau}{6} ; \\
& \mathcal{V}_{12}=\frac{m q_{f}^{2} \omega^{2} \tau}{6}+\frac{m g q_{f} \omega^{2} \tau^{3}}{48} ; \\
& \mathcal{V}_{13}=\frac{m q_{f}^{2}}{2 \tau}-\frac{m q_{f}^{2} \omega \cos (\omega \tau)}{2 \sin (\omega \tau)} ; \\
& \mathcal{V}_{22}=\frac{m q_{f}^{2} \omega^{2} \tau}{6}+\frac{m g q_{f} \omega^{2} \tau^{3}}{24}+\frac{m g^{2} \omega^{2} \tau^{5}}{240} ; \\
& \mathcal{V}_{33}=\frac{m q_{f}^{2} \omega^{2} \tau}{4 \sin ^{2}(\omega \tau)}-\frac{m q_{f}^{2} \omega \cos (\omega \tau)}{4 \sin (\omega \tau)}
\end{aligned}
$$

Da expressão (15), percebemos que a ação chega em um valor estacionário quando $\alpha=1$ e $\beta=0$. Mais uma vez, esse resultado está de acordo com o princípio de Hamilton.

No caso do oscilador harmônico, a função teste $D_{1}$ é dada por

$$
D_{1}=2\left(\mathcal{T}_{22}-\mathcal{T}_{11}+2 \mathcal{V}_{12}-\mathcal{V}_{11}-\mathcal{V}_{22}\right)
$$

Usando (9), (11) e (16) na equação anterior, chegamos no seguinte resultado

$$
D_{1}=\frac{m g^{2} \tau^{3}}{12}\left(1-\frac{\omega^{2} \tau^{2}}{10}\right) \text {. }
$$

Como podemos ver, a função teste $D_{1}$ é positiva para $\tau<\sqrt{10} / \omega$ e negativa para $\tau>$ $\sqrt{10} / \omega$. 
A função teste $D_{2}$ é dada por

$$
D_{2}=\frac{\left(\mathcal{T}_{22}-\mathcal{T}_{11}+2 \mathcal{V}_{12}-\mathcal{V}_{11}-\mathcal{V}_{22}\right)\left(\mathcal{V}_{13}-\mathcal{V}_{11}\right)}{\left(\mathcal{V}_{12}-\mathcal{V}_{11}\right)^{2}}
$$

Usando (9), (11) e (16), obtemos

$$
D 2=\frac{48}{(\omega \tau)^{4}}\left(1-\frac{\omega \tau \cos (\omega \tau)}{\sin (\omega \tau)}-\frac{(\omega \tau)^{2}}{3}\right)\left(1-\frac{(\omega \tau)^{2}}{10}\right)
$$

A função $D_{2}$ deve ser analisada numericamente. Como podemos ver em Fig. 5, $D_{2}$ alterna entre regiões onde seu valor é maior e menor que 1. Quando $\omega \tau<\pi, D_{2}>1 \mathrm{e}$ $D_{1}>1$, logo, dentro desse domínio e se comparado a todos os movimentos virtuais $q_{v}$ da família (6), o movimento $q_{3}$ minimiza $\mathcal{S}_{3}$. Para $\omega \tau>\pi, D_{1}<1$ e $-\infty \leq D_{2} \leq \infty$, logo, o ponto estável de $\mathcal{S}_{3}$ oscila entre ser um ponto de máximo e ponto de sela dentre os movimentos reprentados por (6).

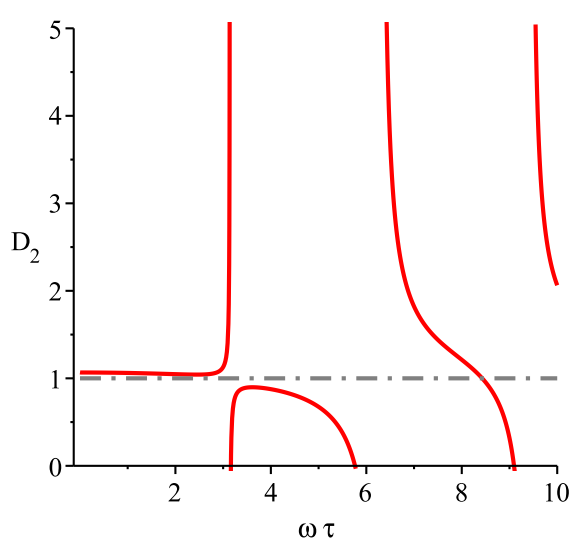

Figura 5: Gráfico de $D_{2}$ em função de $\omega \tau$. A linha traço-ponto representa $D_{2}=1$.

No caso do oscilador harmônico, [3], [4] e [5] indicam que para $\tau<\pi / \omega$, o movimento real do oscilador harmônico minimiza sua ação, ao passo que para $\tau>\pi / \omega$, o movimento real gera um "ponto"de sela. Embora a primeira vista, possamos ter a impressão de que os resultados apresentados nesse artigo não estejam de acordo com esse resulytado, devemos lembrar que a família (6) não abrange todas os movimentos virtuais possíveis.

Tomemos como exemplo, o movimento virtual dado por

$$
q_{e x}=q_{3}\left[1+\kappa \operatorname{sen}\left(\frac{n \pi t}{\tau}\right)\right] .
$$


Nesse caso, a ação é dada por

$$
\mathcal{S}_{3, e x}=\mathcal{T}_{33}-\mathcal{V}_{33}+\kappa^{2} \mathcal{T}_{11}\left[(n \pi)^{2}-(\omega \tau)^{2}\right]
$$

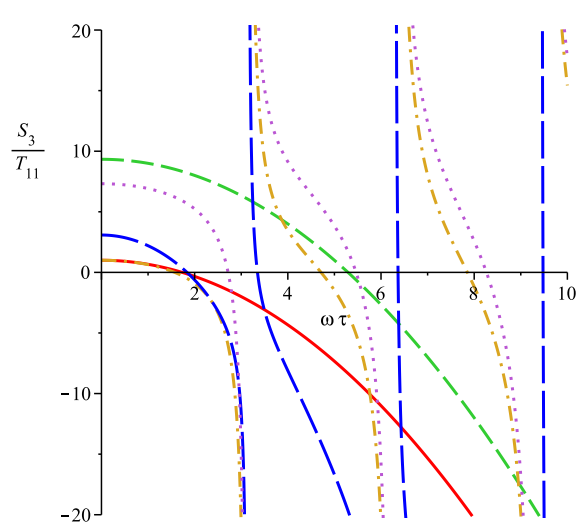

Figura 6: Gráfico de $\mathcal{S}_{3} / \mathcal{T}_{11}$ em função de $\omega \tau$. Usamos $g \tau^{2} / q_{f}=1$ e os mesmos padrões da Fig. 1. A linha pontilhada representa $\mathcal{S}_{3, e x} / \mathcal{T}_{11} \operatorname{com} \kappa=0,2$ e $n=4$.

Na Figura 6, apresentamos os gráficos normalizados de $\mathcal{S}_{3}$ e $\mathcal{S}_{3, e x}$ em função do tempo para diferentes valores de $\alpha$ e $\beta$ e com $n=4$ e $\kappa=0,2$. Como podemos ver, para $0<\omega \tau<\pi, \mathcal{S}_{3}$ apresenta os menores valores quando avaliada em $q_{v}=q_{3}$. Para $\pi<\omega \tau<10, \mathcal{S}_{3}$ avaliada em $q_{v}=q_{3}$ apresenta valores intermediários (ponto de sela), se comparado aos outros casos ilustrados.

Analisando a equação (18), é possível verificar que, para $\omega \tau<n \pi, \mathcal{S}_{3, e x}$ é maior que $\mathcal{S}_{3}$ avaliada em $\alpha=1$ e $\beta=0$. Portanto, é sempre possível encontar um movimento virtual dentro da família (17) que gera uma ação $\mathcal{S}_{3, e x}$ maior que a ação do oscilador harmônico avaliada em seu movimento real. Junto com a análise de $D_{1}$ e $D_{2}$, isso nos garante que, para $\omega \tau>\pi$, o movimento real do oscilador harmônico gera um "ponto"de sela em sua ação.

\section{Conclusão}

Neste artigo, calculamos as ações dos problemas da partícula livre, do lançamento vertical e do oscilador harmônico avaliadas em uma família específica de movimentos virtuais, a combinação linear dos movimentos dos três problemas em estudo. A simplificação em nossa abordagem nos permitiu trabalhar com funções de duas variáveis no lugar de funcionais. Isso, por si só, já trás uma grande vantagem para a compreensão do princípio de Hamilton por parte de estudantes iniciantes em Mecânica Clássica, uma vez que, em 
geral, eles já tem uma boa familiaridade com funções de duas variáveis e nenhum familiaridade com funcionais. Trabalhar com funções de duas variáveis, também nos permitiu traçar gráficos das ações avaliadas em diferentes movimentos virtuais e, consequentemente, identificar e visualizar de forma direta a natureza dos pontos estacionários das ações em estudo.

\section{Agradecimentos}

Gostaríamos de agradecer aos professores Alex Farah Pereira, Felipe Siqueira de Souza da Rosa e Guilherme Guedes de Almeida pelas discussões esclarecedoras. Gostaríamos ainda de agradecer aos avaliadores deste artigo pelas valiosas sugestões.

\section{Referências}

[1] LEMOS, N.A. Mecânica Analítica, São Paulo, Editora Livraria da Física, 2007.

[2] ThORNTON, S.T.; MARION, J.B. Dinâmica Clássica de Partículas e Sistemas, São Paulo, Editora Cengage Learning, 2011.

[3] GRAY, C.G.; TAYLOR, E.F. When action is not least, American Journal of Physics, v. 75, n. 5 , p. 434-458, 2007.

[4] FREIE, W.H.C., A derivada funcional de segunda ordem da ação: investigando minimalidade, maximalidade e ponto de sela, Revista Brasileira de Ensino de Física, v.34, n. 1, 1301, 2012.

[5] MORICONI, M. Condition for minimal harmonic oscillator action, American Journal of Physics, v. 85, p. 633-634, 2017.

[6] STEWART, J. Cálculo - Vol. 2, 6 a edição, São Paulo, Editora Pioneira Thomson Learning, 2009. 